\title{
Antisemitismus und biblische Textkritik bei Paul de Lagarde et al.
}

Am 10.1.2018 zitierte die Hannoversche Allgemeine in dem Artikel „Nimmt der Antisemitismus in Hannover zu?" die Vorsitzende der liberalen jüdischen Gemeinde in Hannover: „Man kann nur abraten, mit Kippa durch die Stadt zu gehen. “1 Das Zitat reflektiert den ständig wachsenden Antisemitismus nicht nur in Deutschland, sondern überall in Europa. Auch und gerade meine Wahlheimat Österreich ist keine Ausnahme.

Warum macht es in dieser Situation Sinn, sich mit Paul Anton de Lagarde und seiner Textkritik zu beschäftigen? Wäre es nicht wichtiger gegen die konkreten Bedrohungen vorzugehen, denen die Juden Europas ausgesetzt sind?

Eine erste Antwort scheint mir offensichtlich. Beides ist wichtig. Das Wiederaufleben des Antisemitismus hat viele Quellen. Unwissen hilft antisemitische Vorurteile $\mathrm{zu}$ verbreiten. Antisemitisches Gedankengut wird nicht nur in offen antisemitischen Schriften, sondern auch in anderen Kontexten kommuniziert, wie z. B. in den wissenschaftlichen Publikationen Lagardes.

Während der Antisemitismus der politischen Publikationen Lagardes, wie etwa seiner Sammlung Deutsche Schriften ${ }^{2}$ unumstritten ist, gilt dies für seine wissenschaftliche Arbeit nicht in gleichem Umfang. Lagarde ist insbesondere in der Forschung an der Textgeschichte und Textkritik der Septuaginta, also der christlichen Sammlung der ältesten griechischen Übersetzungen der Hebräischen Bibel, immer noch hoch angesehen. Seine Forschungen bilden zu Teilen unumstrittene Axiome der heutigen Septuaginta-Forschung. Die forschungsgeschichtliche Arbeit am Werk Paul de Lagardes trägt also eine besondere Verantwortung einer eventuellen Tradierung antisemitischen Gedankenguts durch Lagardes Schriften in der textkritischen Arbeit an der griechischen Fassung des christlichen Alten Testaments und an den jüdischen Ursprüngen dieser griechischen Bibel zu begegnen. ${ }^{3}$

1 http://www.haz.de/Hannover/Aus-der-Stadt/Uebersicht/Hannover-Vertreter-juedischer-Gemeinden-sehen-Antisemitismus-auf-dem-Vormarsch (07.05.2019).

2 Lagarde, Paul de: Deutsche Schriften. Göttingen 1878.

3 Mein Aufsatz ist lediglich an einem Einzelaspekt des antisemitischen Gedankenguts de Lagardes interessiert. Zum Antisemitismus von Paul de Lagarde und seinen zeitgeschichtlichen Hintergründen verweise ich daher auf die Beiträge von Botsch, Gideon u. Werner Treß: Moderner Antisemitismus und Sattelzeit. Das Beispiel Paul de Lagarde (in diesem Band S. 111-126), Puschner, Uwe: Mehr als ein Adjektiv - Völkisch: Bewegung, Weltanschauung, Traditionen (in 
Wie gesagt, an Lagardes Antisemitismus kann kein Zweifel bestehen. Das zeigt schon eine Retrospektive seiner Frau Anna de Lagarde:

Er hat das Judenthum bekämpft, von jeher und bis ans Ende: er wäre nicht der echte Deutsche und der echte Anhänger des Evangeliums gewesen, der er zu sein strebte, wenn er es nicht gethan hätte. ${ }^{4}$

Sein Antisemitismus hat Lagarde schließlich auch zum Gegenstand der Arbeit von Yad VaShem gemacht. Die Yad VaShem Encyclopedia aus dem Jahr 1990 stellt fest, dass Lagardes Deutsche Schriften eine antisemitische Weltsicht reflektieren, die bereits alle Elemente der späteren Nazi-Ideologie beinhaltet. ${ }^{5}$

\section{Antisemitismus damals und heute}

Obwohl an Lagardes Antisemitismus kein Zweifel bestehen kann, scheint es mir wichtig, offenzulegen, welche Antisemitismusdefinition ich in diesem Artikel verwende. Dies ist umso bedeutender, als dass ich in diesem Beitrag auch die judenfeindlichen Polemiken antiker, mittelalterlicher und frühneuzeitlicher christlicher Autoren als antisemitisch charakterisieren werde.

Zum einen halte ich die weit verbreitete Unterscheidung zwischen religiös motiviertem Judenhass als Antijudaismus einerseits und rassistisch motiviertem Judenhass als Antisemitismus andererseits für unangebracht, da selbst zur Nazizeit, aber auch noch heute, etwa bei White Supremacists, religiöse und rassistische Elemente des Judenhasses untrennbar miteinander verschmolzen sind. ${ }^{6}$ Zum anderen scheint es mir wichtig, eine allgemein verbindliche Definition von Antisemitismus zu verwenden, wie sie von der International Holocaust Remembrance Alliance vorgeschlagen und von einer Vielzahl von Regierungen und

diesem Band S. 126-145), Paul, Ina U.: Paul de Lagarde und „die Juden“ (in diesem Band S. 9-29) und Gertzen, Thomas L.: Orientalismus und Antisemitismus. Vergleichende Betrachtungen zu Ernest Renan, Paul de Lagarde, Eduard Meyer und Friedrich Delitzsch (in diesem Band S. 167190).

4 Lagarde, Anna: Paul de Lagarde. Erinnerungen aus seinem Leben für die Freunde zusammengestellt. Göttingen 1894. S. 140.

5 Für den Hinweis auf die Yad VaShem Encyclopedia danke ich der Chefhistorikerin von Yad VaShem, Dina Porat.

$6 \mathrm{Zu}$ einer detaillierteren Begründung siehe Lange, Armin u. Maxine L. Grossman: Jews and Judaism between Bedevilment and Source of Salvation: Christianity as a Cause of and a Cure against Antisemitism. In: An End to Antisemitism! Comprehending and Confronting Antisemitism. Hrsg. v. Armin Lange [u. a.]. Berlin 2019. S. 133-164. 
internationalen Organisationen in der Zwischenzeit anerkannt wurde. Ich spreche hier von der sogenannten Working Definition of Antisemitism: ${ }^{7}$

\begin{abstract}
Antisemitism is a certain perception of Jews, which may be expressed as hatred toward Jews. Rhetorical and physical manifestations of antisemitism are directed toward Jewish or nonJewish individuals and/or their property, toward Jewish community institutions and religious facilities.

Manifestations might include targeting of the state of Israel, conceived as a Jewish collectivity. However, criticism of Israel similar to that leveled against any other country cannot be regarded as antisemitic. Antisemitism frequently charges Jews with conspiring to harm humanity, and it is often used to blame Jews for „why things go wrong.“ It is expressed in speech, writing, visual forms and action, and employs sinister stereotypes and negative character traits.
\end{abstract}

Die Working Definition schließt dabei ausdrücklich auch Formen des klassischen Antisemitismus bzw. Antijudaismus mit ein:

Using the symbols and images associated with classic antisemitism (e.g., claims of Jews killing Jesus or blood libel) to characterize Israel or Israelis. ${ }^{8}$

\title{
2 Lagardes Antisemitismus in der Forschungsgeschichte der Textkritik der Hebräischen Bibel
}

Die forschungsgeschichtliche Literatur zu Lagarde trennt in der Regel peinlich genau zwischen seinen politischen und antisemitischen Veröffentlichungen einerseits und seinen wissenschaftlichen Arbeiten andererseits. Frühe Beispiele dafür sind das Buch von Alfred Rahlfs über Paul de Lagarde, ${ }^{9}$ der sich mit Lagardes Antisemitismus gar nicht auseinandersetzt, und die Grabrede von Ulrich von Wilamowitz-Moellendorff, ${ }^{10}$ der zwischen „nationalgesinnter Romantik“ Lagardes einerseits und „objektivster Urkundlichkeit“ in seiner Philologie und

7 https://www.holocaustremembrance.com/node/196 (30.04.2019).

8 https://www.holocaustremembrance.com/node/196 (30.04.2019).

9 Rahlfs, Alfred: Paul de Lagardes wissenschaftliches Lebenswerk im Rahmen einer Geschichte seines Lebens dargestellt. Göttingen 1928.

10 von Wilamowitz-Moellendorff, Ulrich: Am Sarge von Paul de Lagarde. Rede gehalten im Auftrage der Universität Göttingen am 25. Dezember 1891 von dem zeitigen Prorektor. In: Ders.: Reden und Vorträge. Berlin 1901. S. 90 - 96, 93. 
Textkritik andererseits unterscheidet. ${ }^{11}$ Diese Trennung hat $\mathrm{zu}$ einer positiven Lagarde-Rezeption in vielen Wissenschaftsfächern geführt, die ich für höchst bedenklich halte. ${ }^{12}$

Eine wohltuende Ausnahme ist der im Selbstverlag veröffentlichte Beitrag von Michael Lattke, Paul Anton de Lagarde und das Judentum. ${ }^{13}$ Lattke $^{14}$ weist ganz zu Recht auf das Vorwort von Symmicta (Bd. 2) hin, in dem Lagarde ausdrücklich den Zusammenhang seiner wissenschaftlichen Arbeiten mit seinen Deutschen Schriften betont:

ich bitte alle, welche diesen band lesen wollen, sich vorher durch meine deutschen schriften auf den ton zu stimmen, auf welchen mein leben und meine arbeiten gestimmt sind. ${ }^{15}$

Wie schwierig Lagardes Antisemitismus von seiner wissenschaftlichen Arbeit zu trennen ist, zeigt Lagardes Diskussion der Targum Onqelos Ausgabe von Abraham Berliner. Sein berüchtigter Aufsatz Juden und Indogermanen ${ }^{16}$ ist nämlich sowohl eine Replik auf Abraham Berliners Ausgabe des Targum Onqelos ${ }^{17}$ als auch Teil von Lagardes antisemitisch geprägter Kritik an den Arbeiten von Leopold Zunz und anderen jüdischen Gelehrten. ${ }^{18}$ Wie in vielen Publikationen Lagardes mischen sich in Juden und Indogermanen wissenschaftliche Themen mit Polemik und Judenhass. Die Kritik einer Gruppe jüdischer und anderer Gelehrter genügt

$11 \mathrm{Zu}$ diesem Vorgehen, s. auch Neuschäfer, Bernhard: Alteri saeculo. Paul Anton de Lagardes ,Lebensarbeit‘ an der Septuaginta. In: Die Göttinger Septuaginta: Ein editorisches Jahrhundertprojekt. Hrsg. Reinhard G. Kratz u. Bernhard Neuschäfer. Berlin 2013 (Mitteilungen des Septuaginta-Unternehmens 30). S. 235-264 (242-245).

12 Dazu s. z. B. Lattke, Michael: Paul Anton de Lagarde und das Judentum. Brisbane 2014. https://www.academia.edu/6851085/Paul_Anton_de_Lagarde_und_das_Judentum (30.04.2019). S. 62-64.

13 Lattke, Paul Anton de Lagarde (wie Anm. 12).

14 Lattke, Paul Anton de Lagarde (wie Anm. 12), S. 84.

15 Lagarde, Paul de: Symmicta. 2 Bände. Göttingen 1877-1880; Band 2. S. vii-viii.

16 Lagarde, Paul de: Juden und Indogermanen. In: Ders.: Mittheilungen, 4 Bände. Göttingen 1884-1891. Bd. 2 (1887). S. 262-352.

17 Berliner, Abraham: Targum Onkelos. Herausgegeben und erläutert. Frankfurt a.M. 1884.

18 Lagarde, Paul de: Lipman Zunz und seine Verehrer. In: Ders.: Mittheilungen. Bd. 2 (1887). S. 108 -162. Siehe u.a. auch Kaufmann, David: Paul de Lagarde’s jüdische Gelehrsamkeit. Eine Erwiderung. Leipzig 1887 und Thulin, Mirjam: Wissenschaft und Vorurteil. Die Kontroverse zwischen David Kaufmann und Paul de Lagarde. In: Beschreibungsversuche der Judenfeindschaft: Zur Geschichte der Antisemitismusforschung vor 1944. Hrsg. v. Hans Joachim Hahn u. Olaf Kistenmacher. Berlin 2015. S. 121-148; Thulin, Mirjam: Kaufmanns Nachrichtendienst: Ein jüdisches Gelehrtennetzwerk im 19. Jahrhundert. Göttingen 2012 (Schriften des Simon-Dubnow-Instituts 16). S. 254-283. 
für Lagarde dazu, um eine Verschwörung des Judentums gegen ihn zu postulieren. Auf diese angebliche Verschwörung reagiert er mit folgendem Aufruf:

Es gehört ein Herz von der Härte der Krokodilshaut dazu, um mit den armen ausgesogenen Deutschen nicht Mitleid zu empfinden und - was dasselbe ist - um die Juden nicht $\mathrm{zu}$ hassen, um diejenigen nicht zu hassen und zu verachten, die - aus Humanität! - diesen Juden das Wort reden oder die zu feige sind, dies wuchernde Ungeziefer zu zertreten. Mit Trichinen und Bacillen wird nicht verhandelt, Trichinen und Bacillen werden auch nicht erzogen, sie werden so rasch und so gründlich wie möglich vernichtet. ${ }^{19}$

Solche Äußerungen gingen auch im späten 19. Jahrhundert weit über jegliche Form wissenschaftlicher Polemik hinaus. Juden mit Krankheitserregern zu vergleichen, wirft schon im späten 19. Jahrhundert ebenso unheildrohende Schatten auf die antisemitische Hetze der Nazis voraus wie Lagardes Aufruf zum Genozid, dass Juden ebenso wie Trichinen und Bazillen „so rasch und so gründlich wie möglich vernichtet“" werden müssten. ${ }^{20}$

Wie sehr auch Lagardes Exegese von seinem Antisemitismus geprägt ist, wird an anderer Stelle im gleichen Aufsatz deutlich, wenn er Abraham als intrigant und geldgierig schildert:

Der Mann, nach dem Herr Berliner Abraham heißt, log einst aus Feigheit, da er in richtiger Selbsteinschätzung die Aegypter für Antisemiten hielt, weil die Aegypter es hätten sein müssen: er log dem Könige von Aegypten vor, sein - Abrahams - Eheweib Sara sei seine Abrahams - Schwester. ... Als jener König diese Schwester zur Ehe begehrt und - erhalten hat, tritt alsbald der stille Genosse der Firma in Thätigkeit. Der gutmüthige König schenkt dem Schwager, dem die Wahrheit heilig war, Heerden und Sklaven und Sklavinnen: der stille Theilhaber der Firma schlägt den König wegen eines Ehebruchs, den der Geschlagene nur durch des frommen Patriarchen Schuld begangen hat. Und am Ende wird Abraham, der die Gaben des Schwagers behält, von dem angeblichen Antisemiten freundlich außer Landes geleitet. $^{21}$

Lagardes Forschungen pauschal von seinem Antisemitismus zu trennen, ist angesichts solcher Äußerungen sicherlich verfehlt. Wer Lagarde wissenschaftlich rezipieren möchte, muss vielmehr jede einzelne seiner Studien sorgfältig auf antisemitische Inhalte überprüfen. Wer Lagarde forschungsgeschichtlich würdigen möchte, muss sich nicht nur mit seinem Antisemitismus auseinandersetzen, sondern sollte jede einseitige Hervorhebung seiner philologischen Verdienste vermeiden. Diese Arbeit für das Gesamtwerk Lagardes zu leisten, ist wichtig, da

19 Lagarde. Juden und Indogermanen (wie Anm. 16), S. 339.

20 Lagarde. Juden und Indogermanen (wie Anm. 16), S. 339.

21 Lagarde. Juden und Indogermanen (wie Anm. 16), S. 304. 
Lagarde nicht nur von den Nazis, ${ }^{22}$ sondern auch noch heute ${ }^{23}$ sowohl wissenschaftlich als auch antisemitisch rezipiert wird. Selbst ausgewiesene Experten für die Textkritik der Hebräischen Bibel und des christlichen Alten Testaments wissen dabei nicht immer von Lagardes Antisemitismus!

Das wissenschaftliche Werk Lagardes kann keinesfalls auf seine textkritische Arbeit an der Septuaginta beschränkt werden. Als Philologe und Orientalist war Lagarde z. B. auch in der Arabistik und Koptologie eine wichtige wissenschaftliche Stimme. Was ich hier am Beispiel von Lagardes Rekonstruktion der Textgeschichte der Hebräischen Bibel versuchen möchte, sollte ähnlich auch für die Forschungen Lagardes in anderen Wissenschaftszweigen geleistet werden.

\section{Die jüdische Textgeschichte der Hebräischen Bibel nach Lagarde}

\subsection{Die Anmerkungen zur griechischen Übersetzung der Proverbien}

Jene Errungenschaft, für die Lagarde in der Textkritik und Textgeschichte besonders berühmt ist, ist die Archetyp-Theorie, die er 1863 in seinen „Anmerkungen zur griechischen Übersetzung der Proverbien“ formuliert hat. Der entsprechende Satz lautet:

es ergibt sich also, dafs unsere hebräischen handschriften des alten testaments auf ein einziges exemplar zurückgehen, dem sie sogar die korrektur seiner schreibfehler als korrektur treu nachgeahmt haben. ${ }^{24}$

Es handelt sich hier um eine von vielen Feststellungen, die Lagarde gerne in Einführungen zu seinen Aufsätzen und Büchern trifft und die der dort mit knappen Ausführungen zu untermauern versucht. Es sei hier nur als apropros erwähnt, dass die Idee von einem textlichen Archetyp der Hebräischen Bibel nicht auf Lagarde zurückgeht, sondern schon 76 Jahre vor Lagarde von Johann Gottfried

22 Siehe Sieg, Ulrich: Deutschlands Prophet. Paul de Lagarde und die Ursprünge des modernen Antisemitismus. München 2007. S. 326-353.

23 http://de.metapedia.org/wiki/Lagarde,_Paul_de (30.04.2019).

24 Lagarde, Paul de: Anmerkungen zur griechischen Übersetzung der Proverbien. Leipzig 1863. S. 2. 
Eichhorn formuliert wurde, und zwar in Reaktion auf die kritische Bibel-Ausgabe Benjamin Kennicotts. ${ }^{25}$

Das oben wiedergegebene klassische Lagarde-Zitat wird jedoch selten in den Kontext der Ausführungen Lagardes in seiner Einleitung zu den Anmerkungen zur griechischen Übersetzung der Proverbien gestellt.

Lagarde argumentiert für eine einzige Handschrift als Archetyp des masoretischen Textes der Bibel, um seine Forschungen an der Septuaginta zu rechtfertigen:

es ergibt sich also, dafs unsere hebräischen handschriften des alten testaments auf ein einziges exemplar zurückgehen, dem sie sogar die korrektur seiner schreibfehler als korrektur treu nachgeahmt haben. über diesen archetypus des masoretischen textes würden wir nur durch conjectur hinausgelangen können, wenn uns nicht die griechische version des alten testaments die möglichkeit verschaffte, wenigstens eine schlechte übersetzung eines einer andren familie angehörenden manuscripts zu benutzen. es versteht sich selbst heutzutage leider noch nicht von selbst, dafs die LXX nur in ihrer ursprünglichen gestalt zur kritik unserer masoretischen diaskeuase angewandt werden darf. wollen wir über den hebräischen text ins klare kommen, so gilt es zunächst die urform der griechischen übersetzung zu finden. ehe diese vorliegt, darf die aegyptische recension nicht zur kontrolle der palästinischen benutzt werden. ehe aber eine solche kontrolle vorgenommen worden ist, hat niemand das recht die überlieferung als fest und bekannt anzusehn. ${ }^{26}$

Lagarde hält die beiden hier erwähnten biblischen Texte, den masoretischen Text und die Septuaginta, für verderbt. Als guter Philologe möchte er den ursprünglichen Septuaginta-Text - heute würden wir vom Old Greek sprechen - rekonstruieren, um sich mit Hilfe dieses ursprünglichen Septuaginta-Textes vor-masoretische Stufen des Textes der Hebräischen Bibel zu erschließen. Dieses Vorgehen darf getrost als gute und gängige textkritische Praxis beschrieben werden. Es scheint also, als ob Lagardes berühmte Anmerkung zur ArchetypusTheorie nichts mit seinem Antisemitismus zu tun hat.

25 Eichhorn, Johann Gottfried: Einleitung in das Alte Testament. Bd. 2. 2. Aufl. Leipzig 1787. S. 203 (cf. S. 111 und S. 113): ,daß alle unsere Handschriften zu einer Recension, der masoretischen, gehören“; Kennicott, Benjamin: Vetus Testamentum hebraicum, cum variis lectionibus. 2 Bände. Oxford 1776-1780. Zur Sache siehe Chiesa, Bruno: Appunti di storia della critica del testo dell'Antico Testamento ebraico. In: Henoch 12 (1990). S. 3-14; Tov, Emanuel u. Eugene Ulrich:1.1.1 Textual History of the Hebrew Bible. In: Textual History of the Bible, vol. 1, The Hebrew Bible, part 1a: Overview Articles, Hrsg. v. Armin Lange und Emanuel Tov. Leiden 2016. S. 3-35, 8; Lange, Armin: 1.2.1 Ancient Hebrew-Aramaic Texts: History of Research. In: Textual History of the Bible, vol. 1, The Hebrew Bible, part 1a: Overview Articles. Hrsg. v. Armin Lange u. Emanuel Tov. Leiden 2016. S. 82-112, 96.

26 Lagarde, Anmerkungen (wie Anm. 24), S. 2. 


\subsection{Materialien zur Kritik und Geschichte des Pentateuch}

Gründliche Forschung sollte jedoch voreilige Schlüsse vermeiden. Was die Arbeit an den textkritischen Publikationen Lagardes besonders schwierig macht, ist, dass er seine eigene Theorie zur Textgeschichte der Hebräischen Bibel nie in einer systematischen Darstellung, sondern immer nur in verstreuten Anmerkungen entfaltet hat. Eine solche Anmerkung findet sich in Lagardes Materialien zur Kritik und Geschichte des Pentateuchs: ${ }^{27}$

schon 1863 habe ich in den anmerkungen zur griechischen übersetzung der proverbien nachgewiesen, dafs alle hebräischen handschriften des alten testaments aus einem einzigen exemplar stammen. jetzt veröffentliche ich eine mir damals unbekannte notiz, die als ein historisches zeugniss für jenen damals nur durch combination gefunden satz gelten darf. I 231, 14 ff. wird berichtet die Davididen hätten aus Betaris ein einziges exemplar der hebräischen heiligen schriften mit sich genommen, aus dem dann die andern kopiert worden wären. diese nachricht empfängt eine bestätigung noch durch einen, passim im zweiten hefte dieses werkes besprochenen umstand. die chronologie der patriarchen vor Noe ist im masoretischen texte offenbar gefälscht, und zwar aus dem grunde gefälscht, um die mit hülfe der LXX angestellten berechnungen der christen zu widerlegen, nach denen der messias im jahre 5500 der welt erschienen war. solche fälschungen (welche die kirchenväter so oft den Juden vorwerfen) sind nur denkbar, wenn sie an Einem exemplar vorgenommen werden konnten, aus dem alle übrigen abschriften des textes zu entnehmen waren. Aquila (meine reliquiae graecae $1856 \mathrm{xli}$, meine Clementina 1863 vorrede $13 \mathrm{ff}$ ) hat seine übersetzung offenbar nur angefertigt, damit das im interesse des grimmigsten christenhasses gedokterte hebräische alte testament ja den anständigen ,gebildeten“ nicht unbekannt bliebe, denen hebräisch zu lernen nicht füglich zugemuthet werden konnte.

Lagarde stellt in dieser kurzen Anmerkung mehrerlei fest:

(1) Aus der am Ende des zweiten jüdischen Krieges in den Jahren 134-135 n.Z. von den Römern belagerten Stadt Betar hätte nur eine einzige Kopie der Hebräischen Bibel überlebt. Aus diesem Archetypus seien alle späteren Abschriften der Hebräischen Bibel hervorgegangen.

(2) Die Septuaginta (LXX) zeige, dass dieser hebräische Bibeltext gefälscht sei, um Schriftbeweise für die Messianität Jesu zu unterdrücken.

(3) Lagarde spricht von einem aus „grimmigsten Christenhass gedokterten“ Text der Hebräischen Bibel.

(4) Als Kronzeugen für seine Theorie von jüdischen Bibelfälschern verweist Lagarde auf die Kirchenväter.

27 Lagarde, Paul de (Hrsg.): Materialien zur Kritik und Geschichte des Pentateuchs, fasc. I-II. Leipzig 1867. 1. S. xii. 
Lagardes Betar-Theorie ist durch die Textfunde von Qumran ohne jede Frage widerlegt. ${ }^{28}$ Heute kann kein Zweifel mehr daran bestehen, dass sowohl die hebräische Vorlage der Septuaginta (LXX) als auch der masoretische Text (MT) nicht nur vor dem zweiten jüdischen Krieg, sondern sogar vor der Geburt Jesu existierten. Ob man überhaupt von angemessener wissenschaftlicher Methodik sprechen darf, wenn Lagarde seine These mit einem arabisch-christlichen Text belegt, darf sicherlich angezweifelt werden. Ein solcher arabisch-christlicher Text datiert immerhin mehr als 500 Jahre später als die Belagerung Betars und kann schwerlich auf akkurate mündlich tradierte jüdische Erinnerungen zurückgehen.

Lagardes Deutung der textlichen Differenzen zwischen MT und LXX in der Chronologie des Buches Genesis ist klar von seinem Judenhass geprägt. Seine antisemitische Deutung der Evidenz speist sich dabei aus patristischen antisemitischen Vorurteilen über die Juden als Fälscher der Bibel. Dieses antisemitische Präjudiz wurde nicht nur in Antike und Mittelalter, ${ }^{29}$ sondern auch in der Neuzeit mit steter Regelmäßigkeit wiederholt. ${ }^{30}$ Interessanterweise ist auch der Umgang des Islam mit den jüdischen und christlichen Bibeln vom antisemitischen Topos der Bibelfälschung geprägt. Seit den Anfängen des Islam wird sowohl Juden als auch Christen vorgehalten, sie würden ihre Bibeln fehlinterpretieren bzw. gefälscht haben. ${ }^{31}$ Diese Polemik wird als Tahrif bezeichnet und ist im Islam auch

$28 \mathrm{Zu}$ den Handschriften biblischer Bücher aus der Bibliothek von Qumran, s. Lange, Armin: Handbuch der Textfunde vom Toten Meer, Bd.1: Die Handschriften biblischer Bücher von Qumran und den anderen Fundorten. Tübingen 2009; und die diversen Artikel zur Sache in Lange, Armin u. Emanuel Tov (Hrsg.): Textual History of the Bible. Bände 1.1-3. Leiden 2016-2017.

29 Adler, William: The Jews as Falsifiers: Charges of Tendentious Emendation in Anti-Jewish Christian Polemic. In: Translation of Scripture: Proceedings of a Conference at the Annenberg Research Institute, May 15-16, 1989. Hrsg. v. D. Goldenberg. Philadelphia 1990. S. 1-27; und Resnick, Irven M.: The Falsification of Scripture and Medieval Christian and Jewish Polemics. In: Medieval Encounters: Jewish, Christian and Muslim Culture in Confluence and Dialogue 2 (1996). S. $344-380$.

30 Eine Untersuchung zum antisemitischen Stereotyp der Bibelfälschung in der Neuzeit steht noch aus. Einen ersten Überblick werden ich im folgenden Artikel geben: Lange, Armin: 1.11.1 Polemics: Antisemitism. In: Textual History of the Bible. Bd. 3a: History of Research. Hrsg. Armin Lange und Russell E. Fuller. Leiden, in Vorbereitung.

31 Zur Tahrif-Polemik im Islam, s. Goldziher, Ignaz Ueber muhammedanische Polemik gegen Ahl al-Kitab. In: Zeitschrift der Deutschen Morgenländischen Gesellschaft 32 (1878). S. 341-387; Lazarus-Yafeh, Hava: Intertwined Worlds: Medieval Islam and Bible Criticism. Princeton 1992. S. 19-49; eadem: Tahriff. In: Encyclopaedia of Islam. Bd. 10. 2. Aufl. Hrsg. v. P. Bearman [u.a.]. Leiden 2000. S. 111-112; Adang, Camilla: Muslim Writers on Judaism and the Hebrew Bible: From Ibn Rabban to Ibn Hazm. Islamic Philosophy, Theology and Science. Leiden 1996 (Texts and Studies 23); Pulcini, Theodore: Exegesis as Polemical Discourse: Ibn Hazm on Jewish and Christian Scriptures. Atlanta 1998; Hämeen-Anttila, Jaakko: Corruption of Scripture. In: Encyc- 
heute noch weit verbreitet. Spätestens seit den Arbeiten von Ibn Hazm im Cordoba des 11. Jahrhunderts ${ }^{32}$ versuchten muslimische Gelehrte angebliche jüdische Bibelfälschungen mit philologischen Mitteln nachzuweisen.

Lagarde könnte sowohl durch seine arabistischen als auch seine textkritischen Arbeiten an der Hebräischen Bibel das antisemitische Stereotyp jüdischer Bibelfälschung kennengelernt haben. Er selbst autorisiert seine Bibelfälschungspolemik jedenfalls mit einem pauschalen Hinweis auf „die Kirchenväter.“"33

Weder Lagardes Verweis auf die Kirchenväter noch die lange christliche und muslimische Geschichte der Bibelfälschungspolemik machen diese wahrer, sie erklären aber, wie der antisemitische Topos von den Juden als den Fälschern der Bibel Lagarde erreicht hat. Ich komme darauf unten noch einmal zurück.

\subsection{Die Rezension von Olshausens Beiträgen zur Kritik des überlieferten Textes im Buche Genesis}

Weitere Elemente von Lagardes Theorie zur jüdischen Textgeschichte der Hebräischen Bibel finden sich in seiner Rezension der Beiträge zur Kritik des überlieferten Textes im Buche Genesis von Justus Olshausen. ${ }^{34}$ Lagarde hält in dieser Rezension an dem schon in seinen „Anmerkungen zur griechischen Übersetzung der Proverbien“ und in seinen „Materialien zur Kritik und Geschichte des Pen-

lopedia of the Bible and Its Reception. Bd. 4; Hrsg. v. D.C. Alison [u. a.]. Berlin 2012. S. 826-828; Reynolds, Gabriel Said: On the Qur'anic Accusation of Scriptural Falsification (tahrîf) and Christian Anti-Jewish Polemic. In: Journal of the American Oriental Society 130 (2010). S. 189202; Demiri, Lejla: Tahrif in der vormodernen muslimischen Literatur. In: Das koranische Motiv der Schriftfälschung (tahrif) durch Juden und Christen. Islamische Deutungen und christliche Reaktionen. Hrsg. v. T. Güzelmansur. Regensburg 2014. S. 15-44; Schaffner, Ryan: The Bible through a Qur'ānic Filter: Scripture Falsification (Tahrif) in $8^{\text {th }}$ and $9^{\text {th }}$ Century Muslim Disputational Literature. PhD diss. Ohio State University 2016.

$32 \mathrm{Zu} \mathrm{Ibn} \mathrm{Hazm,} \mathrm{siehe} \mathrm{besonders} \mathrm{Adang,} \mathrm{Muslim} \mathrm{Writers} \mathrm{(wie} \mathrm{Anm.} \mathrm{30);} \mathrm{Pulcini,} \mathrm{Exegesis} \mathrm{as}$ Polemical Discourse (wie Anm. 30).

33 Lagarde: Materialien. 1, S. xii.

34 Paul de Lagardes Rezension von Olshausen, Justus: Beiträge zur Kritik des überlieferten Textes im Buche Genesis. In Lagarde: Symmicta. Bd. 1. S. 50 - 57. Lagardes Rezension erschien ursprünglich in Göttingische gelehrte Anzeigen 39 (1870). S. 1549-1560. Olshausens Beitrag erschien als: Beiträge zur Kritik des überlieferten Textes im Buches Genesis. In: Monatsberichte der königlich preussischen Akademie der Wissenschaften zu Berlin aus dem Jahre 1870. Berlin 1871. S. 380 - 409. Es bleibt anzumerken, dass Lagarde Olshausen in seinem Aufsatz Lagarde: Juden und Indogermanen. S. 312 als seinen Vorgesetzten identifiziert. 
tateuch“ Gesagtem fest. Alle hebräischen Handschriften der jüdischen Schriften würden auf einen Archetypus, also eine Urhandschrift zurückgehen:

Im jahre 1863 habe ich in den anmerkungen zur griechischen übersetzung der proverbien bewiesen (ich betone dies zeitwort), daß alle bisher bekannten handschriften des jüdischen kanons aus Einem archetypus stammen. am nächsten läge es, diesen archetypus für das exemplar der sammlung jenes kanons zu halten, das natürlich officiell war. auffallen würde dann die stellenweise außerordentlich große fehlerhaftigkeit des überlieferten textes. man könnte zu deren entschuldigung freilich sagen, daß der unter Esdras amtierende ausschuß die widersprüche des deuteronomikers etwa gegen den elohisten übersehen hat, also auch wohl fehler in den worten nicht bemerkt haben kann, oder aber, daß er in einem heiligen texte zu corrigieren nicht gewagt, dies letztere wenig wahrscheinlich, da der text erst durch ihn zu einem heiligen wurde: man könnte vielleicht sogar daraus, daß der text eben nicht durchgängig, sondern stellenweise (wie in den beiden ersten büchern regnorum) verderbt ist, auf eine große übereinstimmung der amtlichen ausgabe mit ihren noch nicht amtlichen vorlagen schließen: man hätte also wohl die möglichkeit jene fehlerhaftigkeit unseres textes als gegengrund gegen die ansicht, daß der archetypus unserer handschriften und drucke direkt das exemplar der sammler des kanons gewesen sei, nicht gelten zu lassen. indessen abgesehen von anderen erwägungen, haben wir ein, allerdings durch die mündliche überlieferung eines für geschichtsschreibung und geschichtsauffassung absolut unbegabten volkes hindurchgegangenes, also mit mehr oder weniger unzuverlässigem stoffe vermengtes ausdrückliches zeugnis dafür, daß jenes urexemplar unseres textes der zeit Hadrians angehört. Ich habe es in den materialien zur geschichte und kritik des pentateuchs I 231 veröffentlicht, und bin niemals im unklaren darüber gewesen, daß die dort genannten rabbinen Samaias und Abtalion, über welche Iosephus im anfange des funfzehnten buches nachzulesen ist, wohl nur genannt sind, weil gerade zu ihrer zeit (Waehner II 706) gelehrte aus Betharis eine rolle spielten, mithin auch umgekehrt, wo von Betharis die rede war, leicht ihre namen dem gedächtnisse sich darbieten konnten. ${ }^{35}$

Lagarde wiederholt hier mit dem gleichen Verweis auf den von ihm publizierten christlich-arabischen Text die Idee, dass eine einzige Handschrift aus Betar den Archetyp des masoretischen Texts geformt habe. Über das bereits Gesagte hinaus schreibt er den diesem Archetyp des erhaltenen hebräischen Textes vorausgehenden biblischen Urtext einem Ausschuss aus der Zeit Esras zu.

Auch an der Idee antichristlicher Verfälschungen des biblischen Textes durch die Juden hält Lagarde unvermindert fest - wiederum mit dem Verweis auf die unterschiedlichen Chronologien des MT und der LXX im Buch Genesis.

was aber jene überlieferung als in der grundanschauung richtig erweist, ist der umstand, daß unser text des jüdischen kanons mindestens an Einem punkte gegen das Christentum gerichtete correcturen enthält, mithin sein archetypus aus der christlichen zeit stammen muß, und naturgemäß nicht ganz aus den ersten anfängen derselben stammen kann. ich

35 Lagarde. Rezension (wie Anm. 33), S. 50. 
habe in den materialien I xii auf die chronologie der Genesis als das entscheidende hingewiesen. diese ist nach der meinung, welche jetzt die herrschende ist, oder (besser gesagt) in folge eines aberglaubens ohne jeden beweis für die allein berechtigte gilt, von den Griechen und Samaritanern gefälscht: nach meinem dafürhalten sind die masoretischen zahlen nicht ursprünglich. ${ }^{36}$

Lagarde argumentiert somit, dass sämtliche Unterschiede des erhaltenen hebräischen Textes zum postulierten esranischen Text der Bibel auf die Zeit um den zweiten jüdischen Krieg zurückgehen würden. ${ }^{37}$ Dabei nimmt Lagarde neben antichristlichen Manipulationen auch weitere Fehlerquellen an:

so folgt, daß jenes exemplar von Betharis höchst wahrscheinlich der officiellen ausgabe des Esdras nicht überall entsprach. ich bin geneigt, auch alle die fehler, welche eingebildete gelehrsamkeit in den text getragen hat, dieser periode zur last zu legen. ${ }^{38}$

\subsection{Clementina}

Obwohl Lagardes Anmerkungen zur angeblichen jüdischen Bibelfälschung in seinem oeuvre nicht besonders häufig sind, sollte man sie keineswegs als dem Augenblick geschuldete Idiosynkrasien verstehen. Dass es sich hier um ein tief verwurzeltes antisemitisches Vorurteil bei Lagarde handelt, zeigt seine Charakterisierung Aquilas:

Aquila ...| gehört eine zeit lang, als er von Hadrian gesandt den bau von Aelia Capitolina leitete, unter den schülern der apostelschüler der christlichen gemeinde zu Jerusalem als mitglied an, wird aber wegen seiner astrologischen albernheiten ausgestossen, lässt sich beschneiden und liefert den Juden eine antichristliche deutungen enthaltene übersetzung des alten testaments ins griechische. also ein kaiserlicher prinz, der zuerst zum christenthume, dann nach allerhand verirrungen zum judenthume übertritt. ${ }^{39}$

36 Lagarde. Rezension (wie Anm. 33), S. 51.

37 Zur Sache vgl. auch die kurze Anmerkung bei Neuschäfer, Alteri saeculo (wie Anm. 11), S. 260: „Den masoretischen Text des Alten Testaments hielt er für ein völlig verderbtes, z.T. sogar antichristlich interpoliertes Erzeugnis des pharisäischen Judentums.“

38 Lagarde. Rezension (wie Anm. 33), S. 54.

39 Lagarde, Paul de (Hrsg.): Clementina. Leipzig 1865. S.12-13 = Lagarde, Paul de: Beilage. Einige Bemerkungen über die Verbreitung der in den Clementinen erzählten sagen. In: Ders.: Mittheilungen. Band 1 (wie Anm. 16). S. 36-48, 37. 
Etwas später beschreibt Lagarde Aquila als „greulich jüdelnder übersetzer“ und meint damit wohl die vorher erwähnten antichristlichen Deutungen. ${ }^{40}$

\section{Die Geschichte des hebräischen Textes der Bibel bei Lagarde et al.}

Die Geschichte des hebräischen Bibeltextes nach Lagarde erweckt den Eindruck, als ob er mit Hilfe eines rekonstruierten Originaltextes der Septuaginta den von Esras Ausschuss formulierten ursprünglichen Text der Bibel wiederherstellen wollte. Lagarde lehnt MT als Ausgangspunkt für die Rekonstruktion des von ihm postulierten Esranischen Bibeltextes ab, da er ihn für das Produkt jüdischer Textfälschungen hält. Die Motivation hinter Lagardes Idee einer kritischen Septuaginta-Ausgabe ist somit eine antisemitische.

Aber inwieweit ist Lagardes Geschichte des jüdischen Bibeltextes neu? Ich habe schon oben darauf hingewiesen, dass die Idee einer masoretischen Archetyp-Handschrift aus dem späten 18. Jahrhundert stammt und auf die Arbeiten von Kennicott und de Rossi reagiert. ${ }^{41}$ Neben Eichhorn sind hier besonders Ernst Friedrich Karl Rosenmüller ${ }^{42}$ und Georg Lorenz Bauer (1755-1806) zu nennen. ${ }^{43}$ Sowohl Olshausen ${ }^{44}$ als auch Nöldeke ${ }^{45}$ entwickelten die Idee einer pharisäischen Musterhandschrift bzw. Rezension, von der alle masoretischen Handschriften abstammten, schon vor Lagarde. William Whiston (s.u.) datierte eine angebliche anti-christliche Fälschung der Hebräischen Bibel sogar in die Zeit kurz vor dem zweiten jüdischen Krieg.

40 Lagarde, Clementina, S. 14 = Lagarde, Beilage (wie Anm. 39), S. 39.

41 Cf. Chiesa, Appunti (wie Anm. 24).

42 Rosenmüller, Ernst F.K.: Handbuch für die Literatur der biblischen Kritik und Exegese. Bd. 1. Göttingen 1797. S. 247: „daß sie sämtlich im Ganzen eine Recension darstellen, aus einer Quelle geflossen sind." Siehe auch Rosenmüllers Hinweis in dessen Einleitung zur Neuauflage der 1834 erschienen Tauchnitz-Edition der Hebräischen Bibel: Hahn, A. (Hrsg.): Biblia Hebraica: Ad Optimas Editiones Imprimis Everhardi van der Hooght. Neuauflage. Einleitung von E.F.K. Rosenmüller. Leipzig 1834.

43 Bauer, Georg Lorenz: Salomonis Glassii Philologia Sacra his temporibus accomodata: Post primum volumen Dathii opera in lucem emissum nunc continuata et in novi plane operis formam redacta. Band 2.1: Critica Sacra. Leipzig 1795. S. 396: „omnes codices hebraici V.T., quotquot sunt, sequuntur unam eandemque recensionem, masorethicam nimirum“.

44 Ohlshausen, Justus: Die Psalmen. Leipzig 1853 (Kurzgefasstes exegetisches Handbuch zum Alten Testament 14). S. 17-22.

45 Nöldeke, Theodor: Kanon und Text. In: Ders.: Die alttestamentliche Literatur in einer Reihe von Aufsätzen dargestellt. Leipzig 1868. S. 236-243. 
Lagarde ist damit weder mit seiner Archetypus-Theorie wissenschaftlich produktiv noch geht seine Datierung der angeblichen jüdischen Bibelfälschung auf ihn selbst zurück.

Der Antisemitismus der Textkritiker des 19. Jahrhunderts ist weitgehend unerforscht. Lagarde selber lässt aber keinen Zweifel daran, dass er auch mit der antisemitischen Bibelfälschungs-Verleumdung auf ältere Ideen zurückgreift. Er verweist schließlich selbst auf die Kirchenväter. Lagarde spricht in seinen Materialien zur Kritik und Geschichte des Pentateuchs von „fälschungen (welche die kirchenväter so oft den Juden vorwerfen)“ und vom „im interesse des grimmigsten christenhasses gedokterte(n) hebräische(n) alte testament. “46 Da die Kirchenväter hebräische Texte nicht lesen konnten, bezogen Sie sich mit dieser Polemik im Wesentlichen auf die späteren griechischen Übersetzungen durch Aquila und andere. Das wird schon bei Justin deutlich, der die Bibelfälschungs-Verleumdung als erster belegt: ${ }^{47}$

Fürwahr, nicht schließe ich mich euren Lehrern an, welche die Richtigkeit der von den siebzig Ältesten bei dem ägyptischen König Ptolemäus gefertigten Übersetzung nicht anerkennen, sondern eine eigene Übersetzung versuchen. 2 Ihr sollt wissen, daß sie aus der Übersetzung, welche die Ältesten bei Ptolemäus hergestellt haben, viele Schriftstellen vollständig entfernt haben, in denen klar bewiesen wird, daß von unserem gekreuzigten Jesus verkündet war, er sei Gott und Mensch, er werde gekreuzigt und sterbe. (Justin, Dialog mit Trypho 71.2$)^{48}$

Vor allem solltet ihr, da er etwas vorhergesagt hatte, es nicht wagen, die Prophezeiungen abzuändern oder falsch zu erklären; denn nur ihr selbst werdet den Nachteil haben, Gott aber werdet ihr nicht schaden (Justin, Dialog mit Trypho 84.4).49

Die Polemik, die Juden hätten den Text der Hebräischen Bibel entweder im Original oder in den jüdischen Neuübersetzungen des 2. Jahrhunderts n.Z. gefälscht, um die Wahrheit über die Messianität Jesu und den angeblich gottlosen Charakter der Juden zu unterdrücken, ist in der patristischen Literatur weit verbreitet. Ein weiteres Beispiel findet sich bei dem Judenhasser Johannes Chrysostomus:

46 Lagarde. Materialien. 1, S. xii.

47 Zur Bibelfälschungspolemik in Antike und Spätantike siehe Adler: The Jews as Falsifiers (wie Anm. 29); cf. auch Skarsaune, Oskar: The Question of Old Testament Canon and Text in the Early Greek Church. In: Hebrew Bible/Old Testament: The History of its Interpretation, Vol. 1: From the Beginnings until the Middle Ages (until 1300), Part 1: Antiquity. Hrsg.v. M. Saebø. Göttingen 1996. S. 443-450.

48 Übersetzung nach Häuser, Philipp: Justinus, Dialog: Aus dem Griechischen übersetzt. München 1917 (Bibliothek der Kirchenväter 1.33). S. 117.

49 Übersetzung nach Häuser, Justinus (wie Anm. 48), S. 139. 
Sollten aber die Juden, mit diesem Einwand zum Schweigen gebracht, einen anderen suchen, etwa gegen das, was wir über die Jungfrau gesagt, und uns andere Schrifterklärer entgegenhalten, die sagen, es heiße nicht: Jungfrau, sondern: junges Mädchen, so erwidern wir darauf zunächst, dass der Septuagintatext unter allen wohl mit Recht als der zuverlässigste gilt. Unsere Gegner brachten ihre Erklärung erst nach dem Erscheinen Christi vor, und blieben Juden. Es dürfte also der Verdacht nicht unbegründet sein, dass sie mehr aus Voreingenommenheit so redeten und die Propheten absichtlich entstellten. Die Siebzig aber machten sich hundert und mehr Jahre vor Christus an ihr Werk, und bei ihrer großen Anzahl sind sie über jeden solchen Verdacht erhaben, und sowohl in Anbetracht der Zeit als ihrer Zahl, sowie wegen ihrer gegenseitigen Übereinstimmung verdienen sie wohl viel eher Glauben. (Johannes Chrysostomus, In Matthaeum homiliae 5.2 [5.4]) 50 $^{50}$

Eine Ausnahme von der Regel patristischer Unkenntnis des Hebräischen ist Hieronymus. Obwohl sich die Bibelfälschungs-Polemik in seinem oeuvre nur selten findet, erliegt auch Hieronymus trotz seiner herausragenden Kenntnisse des hebräischen Textes und seiner beeindruckenden philologischen Fähigkeiten der Anziehungskraft dieses antisemitischen Präjudizes. In seinem Kommentar zu Gal 3, 10 vergleicht Hieronymus das Paulus-Zitat von Deut 27, 26 in diesem Vers ausführlich mit der LXX, Aquila, Symmachus, Theodotion, proto-MT, und SP (Samaritanischer Pentateuch). Obwohl das Pauluszitat mit keiner dieser Versionen ganz übereinstimmt, überzeugt die von LXX und SP geteilte Lesart ,alle Worte der Tora“ (כל דברי התורה) Hieronymus, dass Paulus den ursprünglichen Text bewahre. Proto-MT sei dagegen das Produkt einer jüdischen Fälschung, um die Unmöglichkeit jüdischer Torafrömmigkeit $\mathrm{zu}$ verheimlichen:

Frustra igitur illu tulerunt Judaei, ne viderentur esse sub maledicto, si non possent omnia complere quae scripta sunt

Also haben die Juden jene (Worte) zur Täuschung fortgenommen, damit sie nicht als (ein Volk) wahrgenommen würden, das unter einem Fluch sei, wenn sie nicht alles erfüllen könnten was geschrieben steht.

Erst nachdem im Spätmittelalter christliche Gelehrte über ausreichende Hebräisch-Kenntnisse verfügten, wurde der Vorwurf jüdischer Bibelfälschung im breiteren Umfang auf den hebräischen Bibeltext selbst übertragen. Beispiele sind Nikolaus von Lyra (Postilla Litteralis super Biblia 1:3G; 6:277BCD) und Raimund

50 Übersetzung nach Baur, Johannes Chrysostomus: Des heiligen Kirchenlehrers Johannes Chrysostomus Erzbischofs von Konstantinopel Kommentar zum Evangelium des hl. Matthäus. Aus dem Griechischen übersetzt. München 1915 (Bibliothek der Kirchenväter 1.23). S. 88. 
Martini (Capistrum Judaeorum 3.3.29 pp. 2.156, 158; Pugio fidei 2.3.9 pp. 277-279; 3.4.11 pp. $695-696$; 3.3.16.16 p. 850; 3.3.16.27 pp. $859-860 ; 3.3 .16 .32$ p.864). ${ }^{51}$

In der Neuzeit wurde die Bibelfälschungspolemik im Rahmen des protestantisch-katholischen Streites um die Hebraica veritas von katholischer Seite aus wiederbelebt. Ein Beispiel ist Jean Morin (Johannes Morinus; 1591-1659), nach dem der mosaische Autograph der Tora von den Juden gefälscht worden sei, um die Messianität Jesu zu bestreiten..$^{52}$ Ähnliche Ideen finden sich auch bei William Whiston, der ein ganzes Kapitel der Bibelfälschungs-Verleumdung widmet. Die Überschrift des Kapitels lautet wie folgt: ${ }^{53}$

The Jews, about the beginning of the Second Century of the Gospel, greatly alter'd and corrupted their Hebrew and Greek Copies of the Old Testament; and that in many places, on purpose, out of Opposition to Christianity.

Whiston lässt keinen Zweifel, dass diese angeblichen Bibelfälschungen antichristlich motiviert gewesen seien:

They had therefore no other possible Way of stopping the farther Progress of the Gospel among them, in their own Power, but this, of altering and corrupting their own Copies; which were, as one may say, all the Copies, excepting the Samaritan Pentateuch, of the original Hebrew; and thence of altering and corrupting their own Copies of the Septuagint Version also, in such Citations, and in such other Places as might suit their own Designs. ${ }^{54}$

Besonders interessant ist, dass Whiston die angebliche Bibelfälschung der Juden nicht nur kurz vor dem zweiten jüdischen Krieg datiert - also fast in die gleiche

51 Zur Bibelfälschungspolemik im Mittelalter, s. Resnick, The Falsification of Scripture (wie Anm. 27). Zu Raimond Martini und Nikolaus von Lyra s. Resnick, The Falsification of Scripture (wie Anm. 27), S. 374-377; Cohen, Jeremy: The Friars and the Jews. The Evolution of Medieval AntiJudaism. Ithaca 1982. S. 148.

52 Morin, Jean: Exercitationes Ecclesiasticae in Utrumque Samaritanorum Pentateuchum. Paris 1631.

53 Whiston, William: An Essay Towards Restoring the True Text of the Old Testament: And for Vindicating the Citations made thence in the New Testament. London 1722. S. 220-281. Siehe auch Ruderman, David B.: Connecting the Covenants: Judaism and the Search for Christian Identity in Eighteenth-Century England. Philadelphia 2007. S. 53 und Steiger, Johann Anselm: The Development of the Reformation Legacy: Hermeneutics and Interpretation of the Sacred Scripture in the Age of Orthodoxy. In: Hebrew Bible / Old Testament. The History of Its Interpretation. Bd. 2: From the Renaissance to the Enlightenment. Hrsg. v. Magne Sæbø. Tübingen 2008. S. 691-757, S. 751.

54 Whiston, An Essay (wie Anm. 50), S. 223-224. 
Zeit wie Lagarde -sondern ebenso mit den abweichenden Chronologien vom MTGen und LXX-Gen argumentiert. ${ }^{55}$

Dass Whiston mit seiner Bibelfälschungspolemik im 18. Jahrhundert keineswegs in der Gelehrtenwelt isoliert war, beweist kein geringerer als der berühmte Textkritiker der Hebräischen Bibel, Benjamin Kennicott. Kennicott hat gleich auf eine ganze Reihe von Stellen verwiesen, an welchen ein Vergleich des masoretischen Textes mit dem Samaritanischen Pentateuch - also mit der Tora der Samaritaner - zeige, dass die Juden ihren Bibeltext mit voller Absicht gefälscht hätten. ${ }^{56}$ Dabei kann Kennicott sogar unter Rekurs auf den oben zitierten Kommentar des Hieronymus zu Gal 3, 10 von ,the Jews having willfully corrupted their Pentateuch“ sprechen. ${ }^{57}$

Whiston und Kennicott sind in ihrer Verwendung der Bibelfälschungspolemik keine Einzelfälle unter den Gelehrten des 18. und 19. Jahrhunderts. Da Lagarde mehrere Jahre in England verbracht hat, ist es aber gut möglich, dass er Whistons Essay und/oder Kennicotts Arbeiten dort gelesen hat.

Wie dem auch sei, es kann kaum bestritten werden, dass Lagardes antisemitische Deutung der Geschichte des hebräischen Bibeltextes sich aus dem christlichen kulturellen Gedächtnis speist. Anders ausgedrückt, Lagardes Interpretation der biblischen Textgeschichte ist von einem antisemitischen Vorverständnis geprägt, das er unhinterfragt an die Textgeschichte der Hebräischen Bibel anlegt. Lagardes Urteil ist damit selbst in den von mir besprochenen wissenschaftlichen Publikationen nicht von seiner philologischen Analyse geleitet, sondern von den Präjudizien seines Judenhasses.

Dieser Mechanismus, überkommene antisemitische Vorurteile aus dem kulturellen Gedächtnis zu übernehmen, um Juden und Jüdisches mit Ihrer Hilfe zu interpretieren, ist typisch für die antisemitische Wirklichkeitswahrnehmung. Sie trifft auch auf viele andere antisemitische Polemiken Lagardes $\mathrm{zu}-\mathrm{z}$. B. die Idee eines weltverschwörerischen Finanzjudentums. Ich zitiere wiederum Lagarde:

Was vorhanden ist, ist zweierlei. Einmal das Bewußtsein, daß die Juden als Juden uns gänzlich fremd sind, zweitens das Bewußtsein, daß die Juden durch Anwendung von Mit-

55 Whiston, An Essay (wie Anm. 50), S. 225-228.

56 Kennicott, Benjamin: The State of the Printed Hebrew Text of the Old Testament Considered: Dissertation the Second: Wherein the Samar. Copy of the Pentateuch Is Vindicated: The Printed Copies of the Chaldee Paraphrase Are Proved to Be Corrupted: The Sentiments of the Jews on the Heb. Text are Ascertained: An Account Is Given of All the Heb. MSS, Now Known; and Also a Particular Catalogue of CX Heb. MSS, in Oxford, Cambridge, and the British Museum. Oxford 1759. S. 20 -102, bes. S. 47-51; vgl. Ders.: Dissertatio Generalis In Vetus Testamentum Hebraicum. Cum Variis Lectionibus, Ex Codicibus Manuscriptis Et Impressis. Oxford 1780. §24 = S. 47-48.

57 Kennicott, The State of the Printed Hebrew Text (wie Anm. 53), S. 50 -51. 
teln, welche wir für unehrenhaft halten, alles Geld und möglichst vielen Grundbesitz an sich zu bringen wissen, daß sie in Folge der so in ihre Hände gelangten Reichthümer sich in Stellungen drängen, die zu gewinnen ihnen nur dann gegönnt wäre, wenn sie den Takt zeigten, der ihnen in den meisten Fällen fehlt, wenn sie durch hervorragende Leistungen uns vergessen machten, auf welchen Wegen vielfach der Reichthum ihrer Nation erworben ist, wenn sie nicht in Wort und Wesen an ihrer Fremdheit auf Schritt und Tritt erinnerten. ${ }^{58}$

\section{Ergebnis}

Für Lagarde ist der hebräische Text der Bibel, wie er dem Judentum als autoritativ und heilig gilt, das Ergebnis einer bewussten Bibelfälschung. Juden hätten im späten ersten oder frühen zweiten Jahrhundert versucht, alle Hinweise auf die Messianität Jesu aus ihrer Bibel zu entfernen. Mit dieser wirklichkeitsfremden, aber sehr alten antisemitischen Ideologie begründet Lagarde letztendlich auch die Notwendigkeit seiner Forschungen zum Text der Septuaginta. Nur die textkritische Arbeit an der Septuaginta würde es erlauben, über den angeblich von Juden gefälschten hebräischen Bibeltext zu jenem Original zu gelangen, welches Jesus von Nazareth als den Messias ankündigen würde. Zumindest ein Motivator für die textkritische Arbeit Lagardes an der Septuaginta ist somit in seiner antisemitischen Ideologie zu finden.

In der Kürze des mir hier zur Verfügung stehenden Platzes ist es leider nicht möglich, zu fragen, in wieweit Lagardes philologische Detailarbeit ebenso von seinem Judenhass geleitet ist wie die Grundfrage seiner Forschung an der Septuaginta. Ein Beispiel könnte allerdings darauf hinweisen, dass auch seine philologische Detailarbeit nicht immer frei von antisemitischem Hass ist.

In seiner Ausgabe der Clemens von Rom zugeschriebenen Homilien erklärt Lagarde mit Hilfe von antisemitischen Vorurteilen, warum Irenäus von Lyon Aquila fälschlich als den pontinischen Aquila beschreibt: ${ }^{59}$

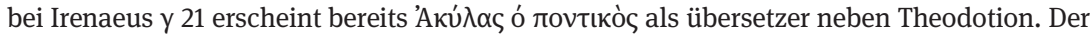

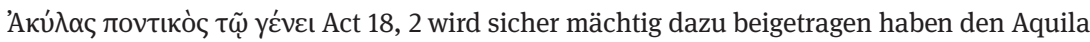
von Pontia in einen Aquila aus Pontus umzuändern. ein so greulich jüdelnder übersetzer wie Aquila musste auch ein greuliches Vaterland haben; der Pontus entsprach allen billigen anforderungen.

58 Lagarde, Paul de: Lipman Zunz und seine Verehrer. In Ders: Mittheilungen. Bd. 2 (1887). S. 108-162, 160-161.

59 Lagarde, Paul de (Hrsg.): Clementina. Leipzig 1865. S. 14 = Lagarde, de, Beilage, S. 39. 
Lagarde behauptet, dass Aquila nicht aus Sinope in Pontus, sondern aus Sinuessa in Pontia stammt. ${ }^{60}$ Eine Hypothese, mit der Lagarde zurecht keinen Glauben fand. Die allgemein übliche Auffassung über die Herkunft des Aquila erklärt Lagarde sowohl auf philologische als auch antisemitische Weise als eine sekundäre Zuschreibung. Philologisch nachvollziehbar ist Lagardes Erklärung, dass Irenäus in seiner Herkunftsbezeichnung von der Erwähnung des pontinischen Aquila aus der Apostelgeschichte beeinflusst wurde. Unter Einfluss der Irenäus sicher wohlbekannten Apostelgeschichte konnte er leicht Pontia mit Pontus verwechseln. Da Pontus aber in der Antike keinen guten Ruf genoss, verbindet Lagarde die angeblich falsche Herkunftsangabe des Irenäus mit antisemitischer Polemik. Irenäus sei in seiner falschen Zuschreibung nicht nur von der Apostelgeschichte in die Irre geführt worden, sondern auch von seinem Judenhass. Daher habe er Aquila als einem schrecklichen ,jüdelnde(n) Übersetzer“ eine ebenso schreckliche Herkunft andichten wollen.

Mein Beispiel zeigt, wie Lagarde auch philologische Einzelbeobachtungen nicht von seinem Judenhass trennen kann. Das Beispiel des pontinischen Aquila ist umso aussagekräftiger, da Irenäus im Vergleich zu anderen patristischen Autoren ein relativ positives Bild vom Judentum hatte und sicher kaum von unreflektiertem Judenhass geleitet war. ${ }^{61}$

Eine solche Einzelbeobachtung ist zwar nicht geeignet, die gesamte philologische und textkritische Arbeit Lagardes als antisemitisch $\mathrm{zu}$ hinterfragen, sie mahnt aber einmal mehr zur sorgfältigen Überprüfung aller philologischen und textkritischen Arbeiten dieses antisemitischen Gelehrten.

\section{Ausblick}

Die Göttinger Lagarde Tagung war nicht nur der Erforschung des wissenschaftlichen Erbes von Paul de Lagarde gewidmet, sondern stand auch unter der Frage, wie mit diesem Erbe zu verfahren sei. Hierzu gehört für mich auch die Frage, wie mit der Person Lagardes umzugehen ist. ${ }^{62}$ In Anbetracht seines antisemitischen Erbes und der Verflochtenheit seiner antisemitischen Ideologie mit seiner wissenschaftlichen Arbeit, scheint es mir unmöglich einen antisemitischen Ideolo-

60 Lagarde, Clementina de (wie Anm. 58), S. 12-15 = Lagarde, Beilage. S. 36-40.

61 Zum Verständnis des Judentums bei Irenäus, siehe Veldt, Mark: Christian Attitudes toward the Jews in the Earliest Centuries A.D. PhD diss. Western Michigan University 2007. S. 157-171.

62 Vgl. etwa „Offener Brief: Keine Huldigung für Antisemiten an der Universität Göttingen,“ https://asta.uni-goettingen.de/offener-brief-keine-huldigung-fuer-antisemiten-an-der-universitaet-goettingen/ (08.05.2019). 
gen des späten 19. Jahrhunderts heute als Vater der Septuagintaforschung oder anderer Wissenschaftsdisziplinen zu ehren. Ich spreche mich im Gegenteil dafür aus, Lagarde jegliche Ehrungen abzuerkennen, da auch Ehrungen eines schon lange verstorbenen Antisemiten geeignet sind, noch heute Judenhass zu legitimieren. Dies würde in letzter Konsequenz bedeuten, auch das Lagarde-Haus in Göttingen umzubenennen. Lagarde sollte lediglich als antisemitischer Vordenker einen „Unehrenplatz“ in unserem kulturellen Gedächtnis einnehmen. Für Lagardes philologische Einzeluntersuchungen, für Lagardes Texteditionen und für sämtliche andere wissenschaftlichen Publikationen von Lagarde sollte gefragt werden, inwieweit diese von seinem Antisemitismus beeinflusst sind, bevor man sie in den wissenschaftlichen Diskurs einbringt bzw. sie in diesem belässt. So wäre es z.B. an der Zeit, die Archetyp-Theorie nicht dem Antisemiten Lagarde, sondern Eichhorn zuzuschreiben, der sie tatsächlich aufgebracht hat. ${ }^{63}$

63 S.o. und Anm. 25. 